\title{
Anterior segment optical coherence tomography angiography to evaluate the peripheral fitting of scleral contact lenses
}

This article was published in the following Dove Press journal:

Clinical Optometry

\author{
Imma Gimenez-Sanchis' \\ Beatriz Palacios-Carmen' \\ Angel García-Garrigós' \\ Javier Cantó-Vañól \\ Antonio J Pérez-Ortega' \\ David P Piñero ${ }^{2}$ \\ 'Opticas ClaraVisión, Ontinyent, Spain; \\ ${ }^{2}$ Department of Optics, Pharmacology \\ and Anatomy, University of Alicante, \\ Alicante, Spain
}

Purpose: The aim of this study was to show the potential applicability of optical coherence tomography angiography (OCTA) for the evaluation of the peripheral fitting of fully scleral contact lenses.

Methods: A pilot study was proposed fitting three different scleral contact lenses (Irregular Corneal Design [ICD]) with different sagittal heights $(4200,4800$, and $5600 \mathrm{~mm}$ ) in a healthy volunteer of 27 years old. We evaluated by means of optical coherence tomography (OCT, DRI Triton) the apical clearance achieved with each of the three lenses fitted. The impact over scleral flow was assessed with the OCTA module of the same device.

Results: The apical clearance was 310,901 , and $1680 \mu \mathrm{m}$ with the scleral lenses of sagittal heights 4200, 4800, and $5600 \mu \mathrm{m}$, respectively. With OCTA, we evaluated the impact of the lens bearing on the conjunctival vascular flow, observing an area of vascular interruption of 0 , 25 , and $75 \%$ with the lenses of 4200,4800 , and $5600 \mu \mathrm{m}$ of sagittal heights, respectively. The vascular interruption was induced in the perilimbar area, suggesting the need of readjusting the limbal clearance zone of the lens.

Conclusion: Fully scleral contact lens fitting may be optimized with the use of OCTA, allowing the practitioner to perform the fitting with better control of the peripheral bearing of the lens on the conjunctival tissue, assessing the impact on vascular structures. This potential use of OCTA must be investigated further in future studies including large samples of eyes.

Keywords: scleral contact lens, optical coherence tomography, OCT angiography, apical clearance, corneal limbus

\section{Introduction}

There has been an increasing interest in fully scleral gas permeable (GP) contact lenses in the last years ${ }^{1,2}$ due to their ability of providing a successful visual rehabilitation in complex cases, such as irregular cornea (primary or secondary) and several dry eye cases, with a very comfortable wear, ${ }^{3-5}$ even in those cases where all other contact lens options fail. ${ }^{6}$ Although the fitting of these contact lenses has been simplified significantly in the last years, there are still some challenges in this process, ${ }^{7}$ including the control of the relationship between lens and conjunctival surface and the level of lens indentation. The introduction of anterior segment optical coherence tomography (OCT) for guiding scleral GP contact lens fitting has allowed the practitioner to achieve a significantly better control of this procedure, especially in the management of the lens vault. ${ }^{8}$ Although the peripheral bearing of the lens is visible in detail with OCT, the evaluation of the impact of the lens bearing on the conjunctival blood flow is still
Correspondence: David P Piñero Department of Optics, Pharmacology and Anatomy, University of Alicante, Crta San Vicente del Raspeig s/n, 03690 San Vicente del Raspeig, Alicante, Spain

Tel +34965909632

Fax +34965903464

Email david.pinyero@ua.es 
based on the observer's subjective perception of the level of conjunctival whitening on slit lamp examination. We report preliminary evidence of the potential usefulness of a new objective method to evaluate the impact of the peripheral bearing of scleral GP contact lenses on conjunctival blood flow, which is based on the use of a new tool included in currently available OCT systems that was initially developed to analyze the retinal and choroidal blood flow, OCT angiography (OCTA). ${ }^{9}$

\section{Methods}

We report the findings of one case fitted with fully scleral GP contact lenses of different sagittal heights. These lenses did not have corneal contact and therefore only rested on the conjunctival-scleral surface. This scleral contact lens fitting was performed in an emmetropic 27-year-old male with a healthy cornea. He was selected for our experiments after being informed and providing written consent according to the tenets of the Declaration of Helsinki.

The Irregular Corneal Design (ICD) 16.5 miniscleral contact lens (Paragon Vision Sciences, Gilbert, AZ, USA) that has four differentiated zones, allowing a correct centration with no corneal touch, such as central clearance zone (CCZ), peripheral CCZ (PCCZ), limbal clearance zone (LCZ), and scleral landing zone (SLZ), was fitted (Figure 1). This contact lens was fitted considering the sagittal height of the cornea height instead of keratometry. Modifications of LCZ and SLZ allow the practitioner to control the level of peripheral contact lens bearing. This scleral contact lens is made up of thermoset fluorosilicone acrylate copolymer derived primarily from siloxane acrylate, trifluoroethyl methacrylate, and methylmethacrylate with a water content of $<1 \%$ (paflufocon D) and the Dk (oxygen permeability) of 100 Fatt (HDS 100 material; Paragon Vision Sciences).

The selection of the trial lens for this case was done following the manufacturer's guidelines. As recommended, we measured the corneal sagittal height for a $10 \mathrm{~mm}$ diameter using the swept source OCT system DRI Triton from Topcon, Tokyo, Japan $(1850 \mu \mathrm{m})$. After this, we estimated the sagittal height of the trial lens by adding 2000 and $300 \mu \mathrm{m}$ of apical clearance to the $10 \mathrm{~mm}$ corneal sagittal height, according to the manufacturer's guidelines. This sum provided the value of $4150 \mu \mathrm{m}$, and therefore, the sagittal height of $4200 \mu \mathrm{m}$ (base curve: $7.67 \mathrm{~mm}$, power: $-2.00 \mathrm{D}$ ) was selected for the first trial lens. Once fitted, we waited for 30 minutes for evaluating the apical vault of the lens by means of the OCT and performing an OCTA analysis. OCTA relies on the interferometric detection of low temporal coherence light back-scattered by moving red

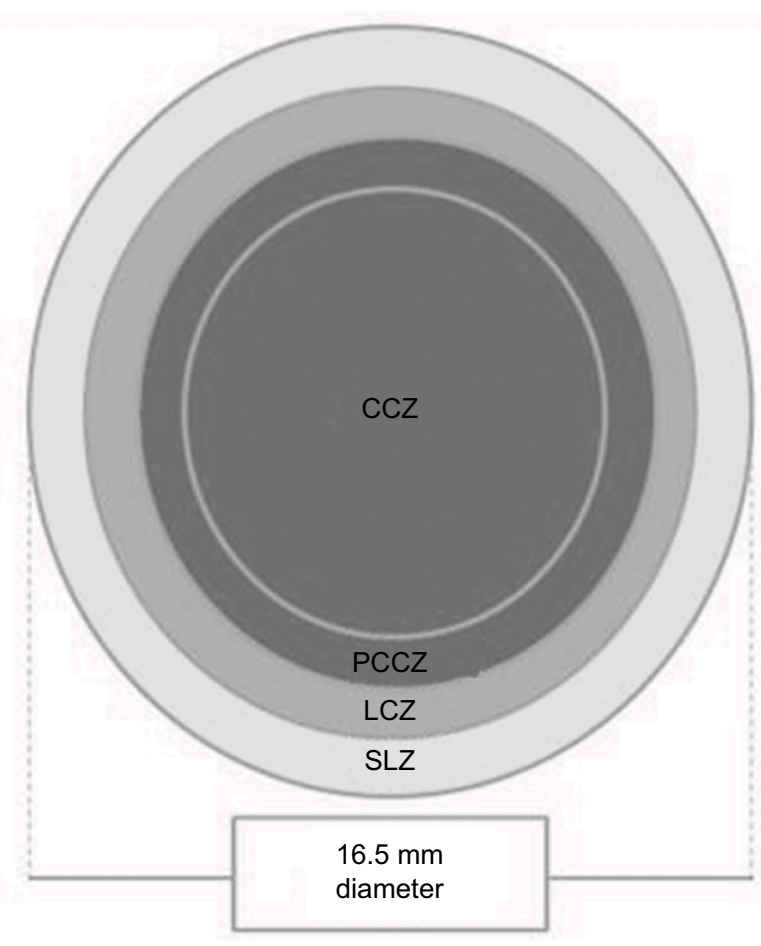

Figure I Diagram showing the design of the contact lens fitted in the case described. Abbreviations: CCZ, central clearance zone; LCZ, limbal clearance zone; PCCZ, peripheral CCZ; SLZ, scleral landing zone.

blood cells. ${ }^{9}$ The resultant B-scan images are analyzed for relative change in tissue reflectivity induced by the cells between two sequential scans. ${ }^{9}$ Currently, this analysis is commonly performed to analyze retinal and choroidal circulation after focusing the system at the retinal plane. ${ }^{9}$ In our experiment, we changed the focus to the sclero-conjunctival surface after adding the lens required by the system for performing anterior segment OCT measurements and selecting the OCTA mode of the instrument. Previous studies have demonstrated that this method for acquiring OCTA images of limbal and conjunctival vasculature provides measurements with substantial consistency. ${ }^{10}$

After all measurements were taken, the lens was extracted and a second lens was fitted 1 hour after with $4800 \mu \mathrm{m}$ of sagittal height (base curve: $6.61 \mathrm{~mm}$, power: $-8.00 \mathrm{D}$ ). The same measurement procedure that followed for the lens of $4200 \mu \mathrm{m}$ was performed. Finally, the same protocol was followed after fitting a lens of $5600 \mu \mathrm{m}$ of sagittal height (base curve: $6.03 \mathrm{~mm}$, power: $-16.00 \mathrm{D})$. For OCTA analysis, a mark was made on the edge of the scleral lens and the area where this mark was resting was analyzed. Thus, the bearing of the same part of the lens was always evaluated. We estimated the level of vascularization density. This evaluation was subjective as no specific analysis tool was available in the software to perform this type of estimation. 


\section{Results}

As expected according to our calculations, the central vault of the scleral lens of $4200 \mu \mathrm{m}$ fitted in the eye was $310 \mu \mathrm{m}$, as measured with the OCT system. After this, we evaluated the peripheral position of the lens, analyzing the adequacy of LCZ and SLZ. The evaluation with the slit lamp suggested an acceptable peripheral bearing of the lens, with no conjunctival whitening. The OCTA analysis, as shown in Figure 2, did not show visible interruption of conjunctival vascular flow.

With the lens of $4800 \mu \mathrm{m}$ of sagittal height, the apical clearance was $901 \mu \mathrm{m}$. With this scleral lens, we were not able to discriminate a significant change in the lens peripheral bearing on slit lamp examination. However, on OCTA examination, some level of interruption of the conjunctival vascular flow was observed, as shown in Figure 3. Specifically, a vascularization density of $75 \%$ was observed in the area of analysis, with a semiperipheral area of vascular flow interruption. This suggested that a change in LCZ would be necessary. An increase in the angle associated with this zone (lift of LCZ) of $\sim 2^{\circ}$ may solve this situation, but we must consider that this will increase the apical lens vault $\sim 50 \mu \mathrm{m}$.

Finally, with the lens of $4800 \mu \mathrm{m}$ of sagittal height, the apical clearance was $1680 \mu \mathrm{m}$. In this case, the higher level of pressure over the corneo-scleral area of the LCZ was visible on slit lamp examination, with a clear negative impact on conjunctival vascular flow observed in OCTA image (Figure 4). The vascular flow interruption zone was $~ 50 \%$ of the analyzed area, suggesting that a significant change in the LCZ would be necessary to solve this indentation. We may recommend an increase of $4^{\circ}$ in the $\mathrm{LCZ}$ angle.

\section{Discussion}

The fitting of fully scleral GP contact lens may be significantly optimized with the use of imaging techniques, such as OCT, allowing the practitioner to know exactly the distance from the lens to the cornea and consequently to characterize the relationship among posterior contact lens surface and anterior corneal profile. ${ }^{8,10,11}$ It should be considered that
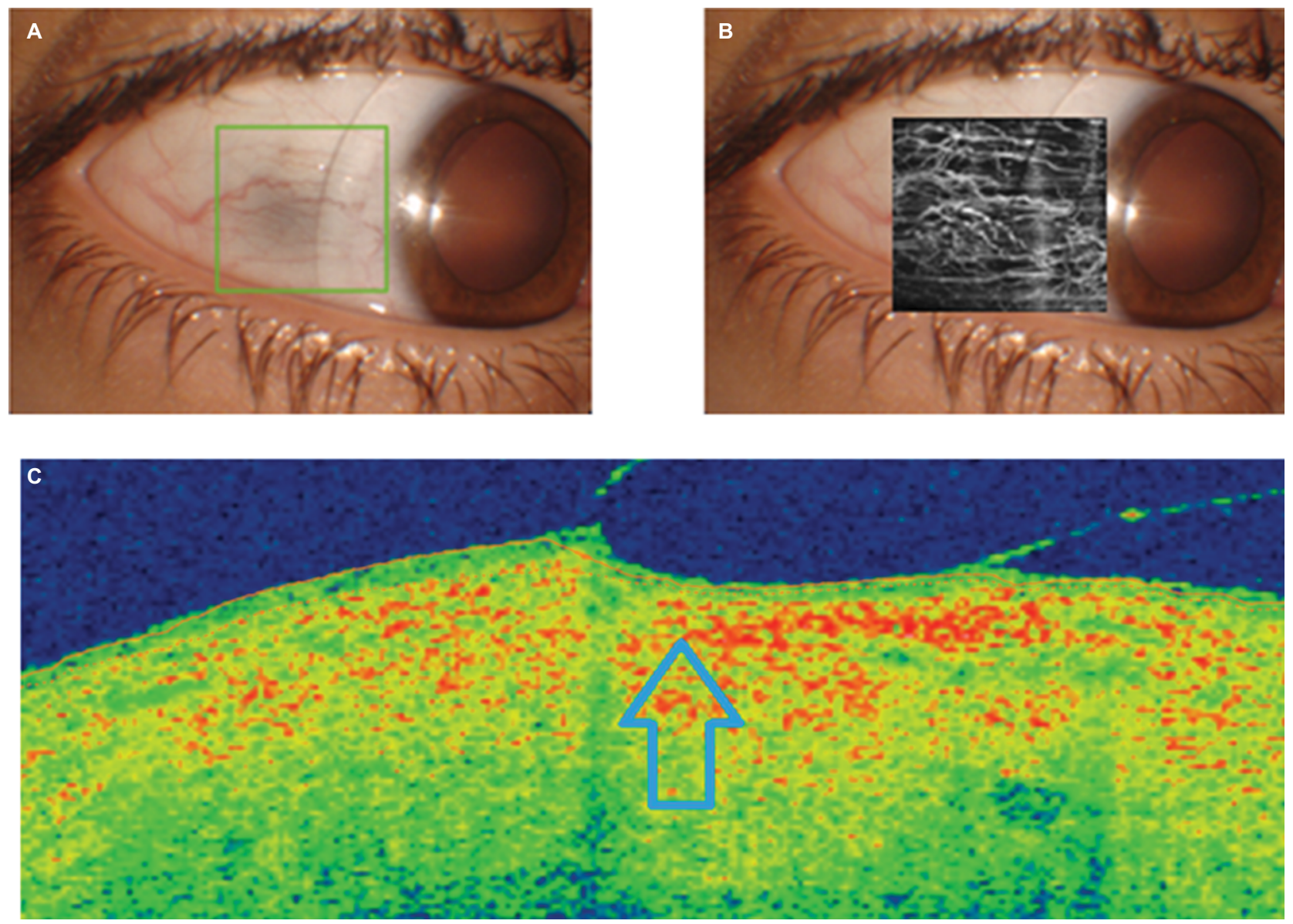

Figure 2 OCT analysis of the peripheral bearing of the scleral contact lens with a sagittal height of $4200 \mu \mathrm{m}$ (A, frontal image; C, OCT section showing the position of the peripheral area of the lens in a specific meridian), including the angiography OCT examination of the impact on the conjunctival vascular flow (B).

Note: Compression area of the peripheral edge of the scleral contact lens is indicated by the arrow.

Abbreviation: OCT, optical coherence tomography. 

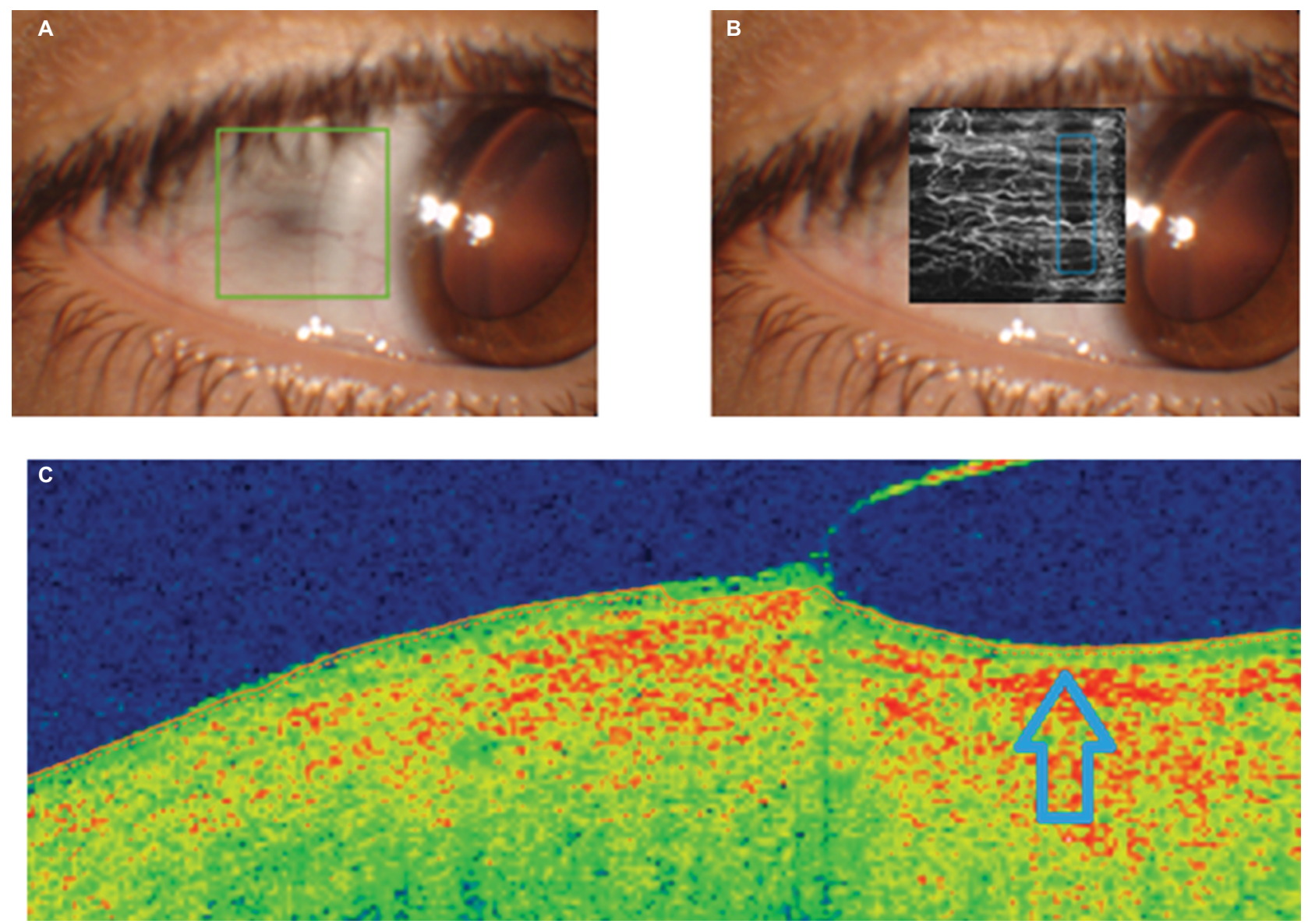

Figure 3 OCT analysis of the peripheral bearing of the scleral contact lens with a sagittal height of $4800 \mu \mathrm{m}(\mathbf{A}$, frontal image; $\mathbf{C}$, OCT section showing the position of the peripheral area of the lens in a specific meridian), including the angiography OCT examination of the impact on the conjunctival vascular flow (B).

Note: Compression area of the peripheral edge of the scleral contact lens is indicated by the arrow.

Abbreviation: OCT, optical coherence tomography.

this relationship changes with the hours of contact lens wear, being recommendable the evaluation of contact lens vault after 4 hours of lens wear. ${ }^{8}$ The control of this relationship is crucial to avoid complications and to minimize the level of corneal thickening associated with the scleral contact lens wear. ${ }^{10}$ However, in spite of the ability of current imaging techniques to analyze the peripheral bearing of the scleral lens on the sclera-conjunctival surface, there are no studies reporting the validity of new options provided by OCT systems for such purpose. In this case report, we show the potential applicability of OCTA to evaluate the impact of the peripheral bearing of scleral lenses on conjunctival blood flow in clinical practice. To our knowledge, this is the first report showing this potential applicability of OCTA.

Our experiment shows the impact of changes in sagittal height of a specific model of fully scleral GP contact lens on conjunctival blood flow by means of OCTA. Specifically, this imaging analysis allowed us to detect how different changes in limbal zone of the scleral lens (LCZ) were necessary for each type of sagittal height. This type of control seems crucial not only to evaluate the peripheral bearing of the scleral contact lens but also to estimate the tolerance of such bearing and the potential risk for medium and long-term complications. It should be considered that anomalies such as conjunctival prolapse and excessive limbal indentation generating hyperemia and discomfort have been reported and appear to be unique sequelae to scleral lens wear. ${ }^{7}$ Therefore, OCTA seems to be a potentially useful tool to consider in the evaluation of the peripheral fitting of fully scleral GP contact lenses, allowing the practitioner to optimize the design of the peripheral regions of such lenses.

In this short communication, we report the fitting of a scleral contact lens with two peripheral areas, such as LCZ and SLZ, allowing theoretically a better control of the limbal clearance and how the lens rests on the conjunctival surface. ${ }^{3,4}$ However, to date, the planning of changes in these areas are only based on subjective biomicroscopic analysis or on OCT images, but without knowing the real impact on the inner structures and vasculature of the superficial signs observed on slit lamp or OCT examination. OCTA is an additional 

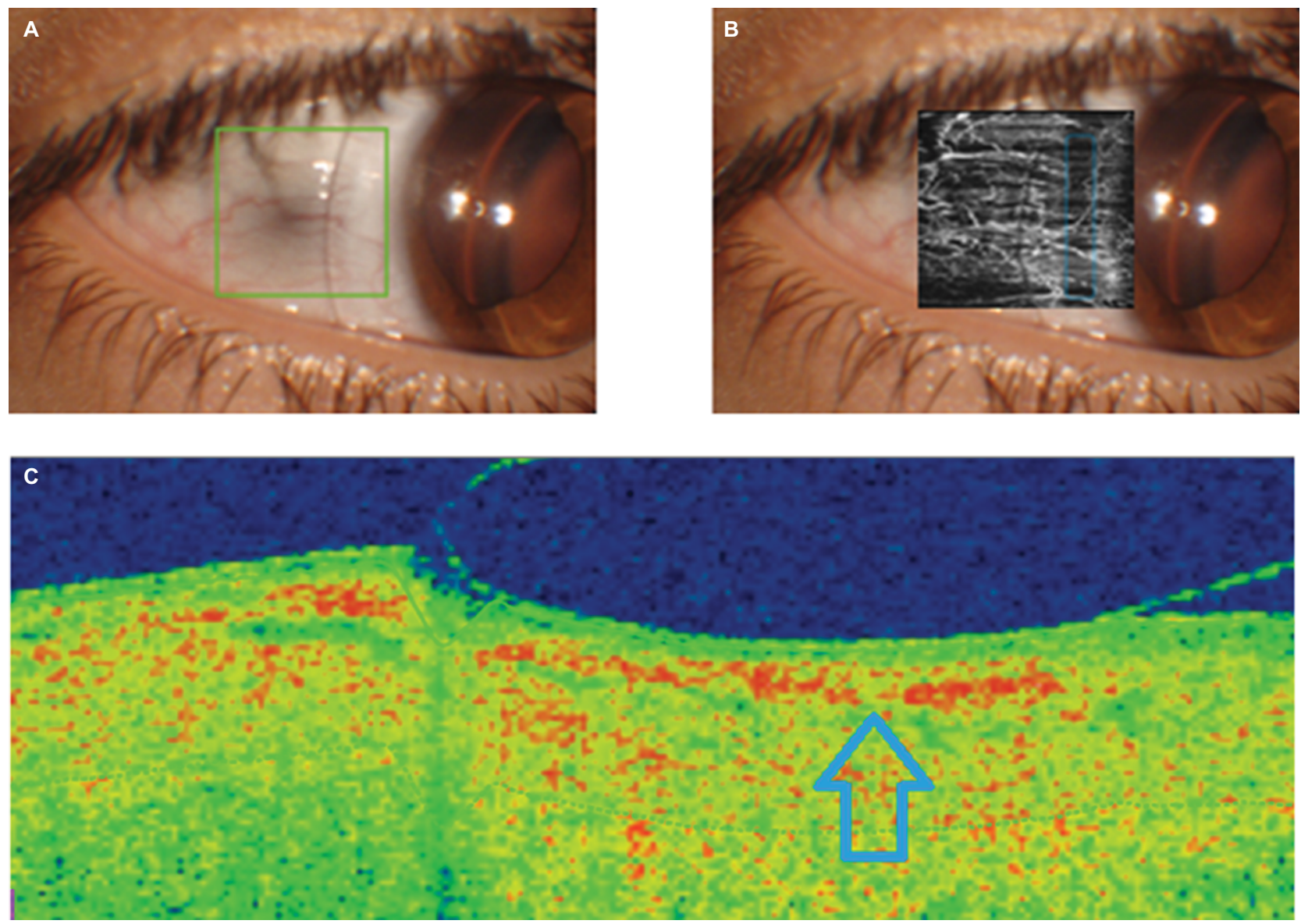

Figure 4 OCT analysis of the peripheral bearing of the scleral contact lens with a sagittal height of $5600 \mu \mathrm{m}(\mathbf{A}$, frontal image; $\mathbf{C}$, OCT section showing the position of the peripheral area of the lens in a specific meridian), including the angiography OCT examination of the impact on the conjunctival vascular flow (B).

Note: Compression area of the peripheral edge of the scleral contact lens is indicated by the arrow.

Abbreviation: OCT, optical coherence tomography.

option to control the impact of the peripheral scleral lens bearing on conjunctival vasculature and therefore to avoid complications derived from an excessive compression of the conjunctival tissue. Vincent et $\mathrm{al}^{12}$ demonstrated that optimally fitted miniscleral contact lenses worn for 3 hours induce significant tissue compression in young healthy eyes, with the greatest thinning observed superiorly, potentially due to the additional force of the eyelid. Most of the morphological changes associated with this compression occur in the conjunctiva/episclera layers, without interference of vascular flow. ${ }^{13}$

This short report shows a potential application of OCTA for scleral lens fitting. However, more studies are required to corroborate the real clinical applicability of this tool, including the analysis of the benefit of using it for optimizing scleral lens fittings as well as the influence of OCT artifacts on the interpretation of the OCT images. In our case, the observed vascular flow interrupt might be caused by optical distortions of the lens edge, ${ }^{14}$ but this seems improbable as the thickness profile was the same in all lenses used. It should be considered that differences in thickness and material might cause different distortions and might potentially have an influence in OCTA analysis. ${ }^{14,15}$ This should be clarified in future studies in which OCTA analysis is performed with different types of scleral contact lenses.

\section{Conclusion}

Fully scleral GP contact lens fitting may be optimized with the use of OCTA that allows the contact lens practitioner to perform the fitting with better control of the peripheral bearing of the lens on the conjunctival tissue and its impact on vascular structures. This potential use of OCTA must be investigated further in future studies including large samples of eyes fitted with scleral contact lenses. Similarly, a grading scale of the level of vascular impact of the scleral bearing of the lens according to OCTA images should be developed to facilitate the use of this technology in clinical practice. Finally, the benefit of OCTA-based fittings should be investigated by comparing their level of complications with those observed in eyes fitted only considering slit lamp signs. 


\section{Acknowledgment}

The author DPP has been supported by the Ministry of Economy, Industry and Competitiveness of Spain within the program Ramón y Cajal, RYC-2016-20471.

\section{Disclosure}

The authors report no conflicts of interest in this work.

\section{References}

1. Van der Worp E, Bornman D, Ferreira DL, Faria-Ribeiro M, Garcia-Porta N, González-Meijome JM. Modern scleral contact lenses: a review. Cont Lens Anterior Eye. 2014;37(4):240-250.

2. Visser ES, Van der Linden BJ, Otten HM, Van der Lelij A, Visser R. Medical applications and outcomes of bitangential scleral lenses. Optom Vis Sci. 2013;90(10):1078-1085.

3. Suarez C, Madariaga V, Lepage B, et al. First experience with the ICD 16.5 mini-scleral lens for optic and therapeutic purposes. Eye Contact Lens. 2018;44(1):44-49.

4. Piñero DP. Fitting of a new design of full scleral contact lens in advanced keratoconus with previous implantation of intracorneal ring segments. Int J Kerat Ect Cor Dis. 2015;4:56-59.

5. Severinsky B, Behrman S, Pery JF, Solomon A. Scleral contact lenses for visual rehabilitation after penetrating keratoplasty: long term outcomes. Cont Lens Anterior Eye. 2014;37(3):196-202.

6. Segal O, Barkana Y, Hourovitz D, et al. Scleral contact lenses may help where other modalities fail. Cornea. 2003;22(4):308-310.
7. Walker MK, Bergmanson JP, Miller WL, Marsack JD, Johnson LA. Complications and fitting challenges associated with scleral contact lenses: a review. Cont Lens Anterior Eye. 2016;39(2):88-96.

8. Rathi VM, Mandathara PS, Dumpati S, Sangwan VS. Change in vault during scleral lens trials assessed with anterior segment optical coherence tomography. Cont Lens Anterior Eye. 2017;40(3):157-161.

9. Kashani AH, Chen CL, Gahm JK, et al. Optical coherence tomography angiography: a comprehensive review of current methods and clinical applications. Prog Retin Eye Res. 2017;60:66-100.

10. Ang M, Sim DA, Keane PA, et al. Optical coherence tomography angiography for anterior segment vasculature imaging. Ophthalmology. 2015;122(9):1740-1747.

11. Esen F, Toker E. Influence of apical clearance on mini-scleral lens settling, clinical performance, and corneal thickness changes. Eye Contact Lens. 2017;43(4):230-235.

12. Vincent SJ, Alonso-Caneiro D, Collins MJ. The temporal dynamics of miniscleral contact lenses: central corneal clearance and centration. Cont Lens Anterior Eye. 2018;41(2):162-168.

13. Alonso-Caneiro D, Vincent SJ, Collins MJ. Morphological changes in the conjunctiva, episclera and sclera following short-term miniscleral contact lens wear in rigid lens neophytes. Cont Lens Anterior Eye. 2016;39(1):53-61.

14. Wolffsohn JS, Drew T, Dhallu S, Sheppard A, Hofmann GJ, Prince M. Impact of soft contact lens edge design and midperipheral lens shape on the epithelium and its indentation with lens mobility. Invest Ophthalmol Vis Sci. 2013;54(9):6190-6197.

15. Sorbara L, Simpson TL, Maram J, Song ES, Bizheva K, Hutchings N. Optical edge effects create conjunctival indentation thickness artefacts. Ophthalmic Physiol Opt. 2015;35:283-292.
Clinical Optometry

\section{Publish your work in this journal}

Clinical Optometry is an international, peer-reviewed, open access journal publishing original research, basic science, clinical and epidemiological studies, reviews and evaluations on clinical optometry. All aspects of patient care are addressed within the journal as well as the practice of optometry including economic and business analyses. Basic and clinical

\section{Dovepress}

research papers are published that cover all aspects of optics, refraction and its application to the theory and practice of optometry. The manuscript management system is completely online and includes a very quick and fair peer-review system, which is all easy to use. Visit http://www.dovepress. com/testimonials.php to read real quotes from published authors. 